\title{
Economic optimization and evolutionary programming when using remote sensing data
}

\author{
Roman Shamin ${ }^{1, *}$, Gabriel Enrike Alberto ${ }^{1}$, Ayzhana Uryngaliyeva ${ }^{1}$ and Aleksandr Semenov ${ }^{1}$ \\ ${ }^{1}$ People's Friendship University of Russia, 117198, Moscow, Russia
}

\begin{abstract}
The article considers the issues of optimizing the use of remote sensing data. Built a mathematical model to describe the economic effect of the use of remote sensing data. It is shown that this model is incorrect optimisation task. Given a numerical method of solving this problem. Also discusses how to optimize organizational structure by using genetic algorithm based on remote sensing. The methods considered allow the use of remote sensing data in an optimal way. The proposed mathematical model allows various generalizations for optimization of decision making in the presence of remote sensing data. The approach associated with evolutionary programming is an effective solution when optimizing economic structures in the presence of remote sensing data.
\end{abstract}

\section{Introduction}

When using remote sensing data of the Earth there are various the problem of economic optimization of the use of these data [1]. This problem has various causes [2]. First, the use of satellite imagery and thematic processing can be economically costly. In addition, in this case, we find that the economic effect has a certain damping, since the information derived from satellite imagery is quickly becoming obsolete. Second, the use of satellite images in monitoring of projects may lead to structural changes in economic planning. In this case, there occurs a problem of evolutionary programming to assess structural changes when using the data of space images. The close topic is discussed in [3-4].

In the present work we consider two economic goals: optimizing the use of satellite images during the implementation of projects and the method of evolutionary programming to optimize economic structures. The research is based on works [5-8].

\section{Optimization of economic efficiency}

We consider a mathematical model of space imagery on the enterprise. The purpose of this model is the calculation of the optimal use of satellite images in such a way as to maintain a predetermined level of effectiveness in the use of space services.

We will consider some venture $\mathrm{P}$. The management of these enterprises is to use space images. The aim of business management is a generalized indicator of efficiency of enterprise activity. Under performance, you can understand the different indicators, for example: profit; the level of innovativeness; competitiveness and etc.
We introduce the corresponding notation. We will consider a mathematical model with continuous time, which we will denote by $t$.

The distribution of the use of space services, we will describe the function $u(t)$, while we assume that this function is a linear combination of generalized functions, because of its economic sense - the intensity of the use of space services is the impulse function.

Performance indicator we will denote by $I(t)$. On the economic meaning of the function $u(t)$ and $I(t)$ are nonnegative. To specify the relationship of the functions $u(t)$ and $I(t)$ must enter the transfer function $K(t)$, which will show the result of the contribution to the impact function $I(t)$ under the pulsed action of the function $u$ at time $t=0$. Using the transfer function, the relationship between the function of the use of space services and performance possible to record in the form of integral equations.

$$
I(t)=\int_{0}^{t} K(t-s) u(s) d s
$$

The transfer function has the following properties

1) $K(t) \geq 0$

2) $K(t)=0, \mathrm{t} \leq 0$

3) $K(t) \rightarrow 0, \mathrm{t} \rightarrow \infty$

The first property shows that the impact of the use of space services for the performance cannot be negative. Of course, in some real situations, this property can be violated, but in our model, we believe that this influence cannot be negative. The second property shows that the past does not depend on the future. This is a natural condition that the use of satellite images only affects the future but not the past. The third property reflects the fact that the effectiveness of the use of space images has a finite duration of the performance, and over time their influence is eroding. kind.

\footnotetext{
Corresponding author: roman@,shamin.ru
} 


\section{The optimization problem}

As we have noted, in economic situations, we must consider not a continuous function of space services, and pulse function. We will consider the use of space snapshots at specific points in time

$$
0<t_{1}<t_{2}<\ldots<t_{\mathrm{N}} \text {. }
$$

In each of the moments $t_{\mathrm{k}}$ the level of use of satellite services will be denoted using $u_{k}$. Using the formalism of $\delta$-functions, the impulse function of the use of space services can be written as follows.

$$
u(t)=\sum_{k=1}^{N} u_{k} \delta\left(t-t_{k}\right) .
$$

Substituting this function in integral equation (1), we get the following equation.

$$
I(t)=\sum_{k=1}^{N} K\left(t-t_{k}\right) u_{k}
$$

Suppose we have a desired level of performance of our company, which is expressed as a function $\mathrm{J}(\mathrm{t})$. Then the problem reduces to finding such values

$$
u_{1}^{*}, u_{2}^{*}, \ldots, u_{N}^{*} \text {. }
$$

After the substitution these values in equation (2), we get the identity

$$
J(t)=\sum_{k=1}^{N} K\left(t-t_{k}\right) u_{k}^{*}
$$

From a mathematical point of view this problem is incorrect because it does not have an exact solution, may not be the only solution, and it can be unstable with respect to perturbations

\section{The method of calculating an optimal plan for use of space services}

We introduce the next values

$$
u_{1}, u_{2}, \ldots, u_{\mathrm{N}} \text {. }
$$

These values we call the plan of use of space services

To optimize the plan that we are thus to minimize the following value

$D\left(t, u_{1}, \ldots, u_{N}\right)=\int_{0}^{T}\left|I(t)-J\left(t, u_{1}, \ldots, u_{N}\right)\right|^{2} d t \rightarrow \min$ Thus, we have a problem of minimizing functions of $\mathrm{N}$ variables.

As we have already noted, this task is incorrect, therefore, for numerical calculations it is necessary to use regularization by Tikhonov. To do this, we modify the original problem by adding to $\mathrm{D}$ a new function with a small parameter.

$$
D\left(t, u_{1}, \ldots, u_{N}\right)+\alpha \Omega\left(u_{1}, \ldots, u_{N}\right) \rightarrow \min .
$$

Here $\alpha>0$ is a small parameter, and the function $\Omega$ is called a stabilizer, which is calculated by the following formula

$$
\Omega\left(u_{1}, \ldots, u_{N}\right)=\sum_{k=1}^{N} u_{k}^{2} .
$$

The use of the stabilizer allows to regularize the original problem.

For the numerical determination of the optimal plan is to use the method of swarming particles, which is effective for multidimensional optimization for nonsmooth functions.

Consider a model example of optimizing the use of space services. We will consider funding for $\mathrm{N}=10$. We consider the situation where we need to ensure the performance of

dimensionless quantities.
As of the transition function consider the following function.

$$
K(t)=\frac{t}{1+t^{2}} .
$$

It is easy to see that this function satisfies the necessary conditions. In addition, it can be shown that this function reflects the economic effect of the use of space services.

We give the results obtained. Optimal use of space services is shown in Fig. 1.

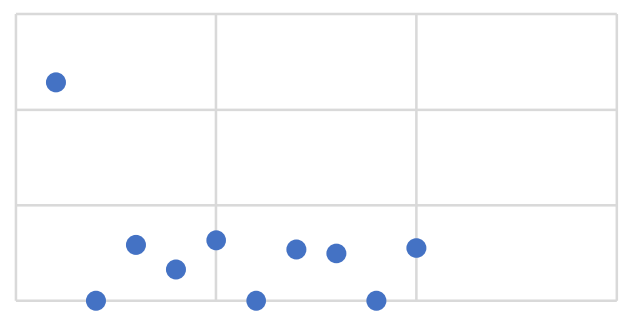

Fig. 1. The optimal plan.

Accordingly, the graph of performance is shown in Fig. 2.

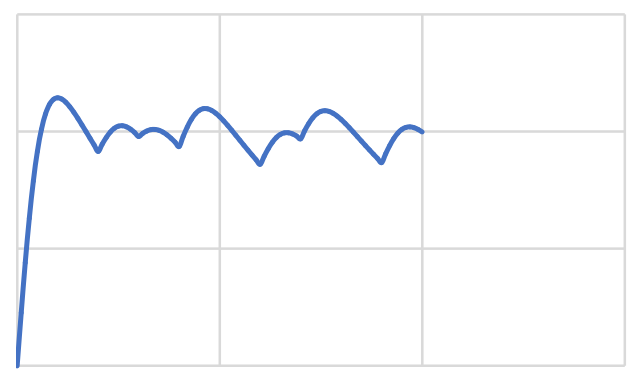

Fig. 2. The performance.

You can see that the proposed plan for the use of space services leads the performance to the desired values, and then supports the desired mode of performance.

\section{The use of the evolutionary method in the task of using space services}

Consider the task of optimizing the structure of enterprise on the basis of the results of the use of space services. This problem can be solved by using evolutionary methods. Let the considered enterprise has a complex structure, which was considered as a directed graph

$$
G=<V, E>\text {, }
$$

where $\mathrm{V}$ is the vertex set of the graph and $\mathrm{E}$ is the directed arcs. In the vertexes there may be structural unit of the enterprise, and arcs of the graph Express the subordination of these structural units in the organization.

A fundamental point for the use of economicmathematical models of optimization is determining the objective function. We consider the following objective function.

$$
H=H(G, \xi)
$$


The function $\mathrm{H}$ accepts numeric values which make sense of economic efficiency of the enterprise. Units of measure this efficiency are irrelevant, because the evolutionary optimization methods do not depend on the actual values of the optimized functions.

Here as the vector $\xi$, we consider the random parameters that affect efficiency.

We introduced a function depends essentially on the own structure of the organization, i.e., from the selected graph G.

The scheme of using the evolutionary method is the application of genetic algorithms to optimize functions of H. In this case, we vary the graph G. This graph is the structure of your organization.

As an elementary change of the graph we will consider the following operations on directed graphs:

- adding vertices;

- delete vertices;

- adding arcs;

- deletion of arcs.

These operations we will consider as basic changes of the graph. These changes are application to an existing structure (the graph $\mathrm{G}$ ) one of these operations.

When used in practice, evolutionary methods of optimization of structure of the enterprise, questions arise regarding the calculation function of efficiency relative to the proposed structure (the graph $\mathrm{G}$ ). To resolve this issue, you can use two fundamental methods - simulation;

- practical application of the changes.

Simulation is a classical way of evaluating the effectiveness of the organization. An important advantage of this method is the fact that we can carry out this estimate "on the computer" without the involvement of material resources and risks. However, this approach is limited by the quality of the simulation, since many factors influence of organizational structure on efficiency are not continuous parameters, i.e. for a slight change in the structure, the efficiency can change dramatically. Thus, the construction of an adequate simulation model is difficult.

Another approach is more radical because it implies that the changes in the company structure implemented, and then it turns out the change (improvement or worsening) performance of the enterprise. Of course, this approach can be implemented is not always, therefore, requires a certain amount of moderation when you change the structure of the enterprise. At the same time, it should be noted that to calculate the real values of the function $\mathrm{H}$ may require lots of time.

Recently, more and more are becoming the methods of artificial intelligence in problems of Economics and management. The use of deep machine learning in conjunction with the construction of expert systems and knowledge bases will allow us to find more accurate methods of evaluating the effectiveness of the structure of the enterprise.

\section{Conclusion}

The paper presents an original mathematical model for performance evaluation of space imagery data application for enterprises. With this extreme model is formulated an optimization problem to describe the optimal scheme of using satellite images for maintaining a given level of productivity of the enterprise.

It is shown that the obtained extremal problem is incorrect problem, therefore, for the computational procedures necessary to use regularization incorrect extremal problems by Tikhonov.

Given a model example of calculation of optimal use of satellite images, which shows a considered economicmathematical model.

Considered a model of optimization of structure of the enterprise based on data derived from remote sensing. This model is based on evolutionary programming.

The paper/article was prepared with the financial support of the Ministry of Education of the Russian Federation in the framework of the scientific project No. 14.575.21.0167 connected with the implementation of applied scientific research on the following topic: «Development of applied solutions for processing and integration of large volumes of diverse operational, retrospective and the thematic data of Earth's remote sensing in the unified geospace using smart digital technologies and artificial intelligence».

\section{References}

1. A.N. Tikhonov, A. Goncharsky, V.V. Stepanov, A.G. Yagola, Numerical Methods for the Solution of Ill-Posed Problems (Springer, 1995)

2. M. Cavazzuti, Optimization Methods (SpringerVerlag Berlin Heidelberg, 2013)

3. P.L. Gurevich, R.V. Shamin, S.B. Tikhomirov. SIAM J. Math. Anal, 45, 1328 (2013)

4. R.V. Shamin, N.V. Bondarchuk, A.V. Fomina, International Journal of Pure and Applied Mathematics, 118, 691 (2018)

5. R.V. Shamin, A.A. Chursin, L.A. Fedorova. European Research Studies Journal, 2039 (2017)

6. C.W. Groetsch, Inverse problems in the mathematical sciences (Braunschweig, Vieweg, 1993).

7. H.W. Engl, M. Hanke, A. Neubauer, Regularization of Inverse Problems (Dordrecht, Kluwer Acad. Publ., 1996)

8. R.V. Shamin, A. Yudin, K. Kuznetsov, A. Kurkin, D. Tyugin, Methods and algorithms of freak wave detection in the coastal zone, In Proceedings of the Twelfth International Conference on the Mediterranean Coastal Environment MEDCOAST, pp. 825-833 (2015) 